\title{
Bistable Output from a Coupled-Resonator Vertical-Cavity Laser Diode
}

A.J. Fischer, K.D. Choquette, W.W. Chow, A.A. Allerman, and K.M. Geib

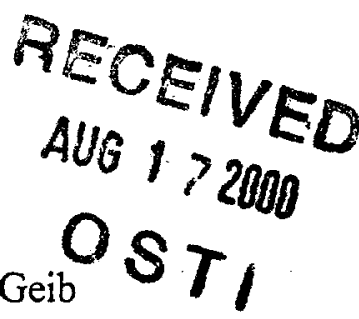
Center for Compound Semiconductor Science and Technology, Sandia National Labs, Albuquerque, NM 87185

\begin{abstract}
We report a monolithic coupled-resonator vertical-cavity laser with an ion-implanted top cavity and a selectively oxidized bottom cavity which exhibits bistable behavior in the light output versus injection current. Large bistability regions over current ranges as wide as $18 \mathrm{~mA}$ have been observed with on/off contrast ratios of greater than $20 \mathrm{~dB}$. The position and width of the bistability region can be varied by changing the bias to the top cavity. Switching between on and off states can be accomplished with changes as small as $250 \mu \mathrm{W}$ to the electrical power applied to the top cavity. Theoretical analysis suggests that the bistable behavior is the response of the nonlinear susceptibility in the top cavity to the changes in the bottom intracavity laser intensity as the bottom cavity reaches the thermal rollover point.
\end{abstract}

Keywords: Vertical-Cavity Surface-Emitting Laser, VCSEL, Coupled-Cavity, Bistability. 


\section{DISCLAIMER}

This report was prepared as an account of work sponsored by an agency of the United States Government. Neither the United States Government nor any agency thereof, nor any of their employees, make any warranty, express or implied, or assumes any legal liability or responsibility for the accuracy, completeness, or usefulness of any information, apparatus, product, or process disclosed, or represents that its use would not infringe privately owned rights. Reference herein to any specific commercial product, process, or service by trade name, trademark, manufacturer, or otherwise does not necessarily constitute or imply its endorsement, recommendation, or favoring by the United States Government or any agency thereof. The views and opinions of authors expressed herein do not necessarily state or reflect those of the United States Government or any agency thereof. 


\section{DISCLAIMER}

Portions of this document may be illegible in electronic image products. Images are produced from the best available original document. 
Semiconductor lasers exhibiting bistable output behavior are attractive for applications involving optical signal processing, high density optical memory, and optical interconnects. Bistable semiconductor lasers have many advantages such as low switching powers, optical gain, and high on-off state contrast [1]. There is much interest in demonstrating bistable operation in vertical-cavity surface-emitting lasers (VCSELs) especially in the form of compact two dimensional arrays which will enable massively parallel optical processing applications. Bistability in VCSELs has been reported due to index changes under optical injection [2], transverse mode hopping [3], saturable absorption $[3,4]$, and polarization state changes [5]. In addition, cleaved-coupled-cavity edge emitters have shown interesting bistable operation [6].

In this paper, we demonstrate bistable behavior in the light-current curve of a coupledresonator vertical-cavity laser (CRVCL). CRVCLs have already shown many interesting and useful phenomena including optical modulation [7], Q-switched operation [8], picosecond pulse generation [9], and dual wavelength operation [10]. An important application for bistable vertical-cavity lasers is in the area of optical data encoding where the bistability leads to high contrast switching at very low electrical switching powers. The bistability regions reported here are much larger than with other laser structures and are positioned at the peak of the lasing output power.

As shown in Fig. 1, the coupled resonator is composed of a bottom p-type distributed Bragg reflector (DBR) with 35 periods, a middle n-type DBR with 11.5 periods, and a top p-type DBR with 22 periods. The top and bottom one-wavelength cavities each contain five $8 \mathrm{~nm}$ GaAs quantum wells. The device was fabricated using a two-tier etch with two metal ring contacts on the top as well as a back side contact. The current can be independently injected into either cavity such that the top cavity can be biased as a saturable absorber. A proton implant was used for current injection in the top cavity while an oxide aperture was used for the bottom cavity. 
Devices were fabricated with and without the proton implant for comparison. As discussed below, devices with an implant aperture in the top cavity could be switched at significantly lower electrical powers.

Fig. 2 (a) shows bistable operation for several different bias currents applied to the top cavity. The output power is greater than $7 \mathrm{~mW}$ and the bistability region extends for more than $18 \mathrm{~mA}$ in current. This device is able to lase with no applied bias current to the top cavity and thus overcome the absorption in the top cavity active region. With increasing bias current to the top cavity, the bistability region narrows and shifts to higher currents. In addition, the lasing threshold decreases and the output power increases with increasing top cavity bias. At very large biases applied to the top cavity, the bistability region can even disappear. These observations are consistent with a lowering of the absorption in the top cavity as the bias in increased. Fig. 2 (b) shows bistability data for another device with a higher threshold and slightly lower output power of $4.8 \mathrm{~mW}$. This device would not lase unless at least $1.5 \mathrm{~mA}$ of current was applied to the top cavity. For this device, lasing abruptly stops closer to the peak of the laser output and exhibits a narrower bistability region. There is also a very abrupt lasing threshold for the $1.5 \mathrm{~mA}$ bias curve (denoted by the arrow in Fig. 2 (b)), which indicates the point at which the absorption in the top cavity is saturated and lasing is possible.

One of the most interesting features of this data is the abrupt shut-off observed at high oxide cavity currents. A VCSEL typically shows a smooth thermal shut off due to a misalignment of the gain and the cavity resonance at higher temperatures. Since the device abruptly transitions from multimode lasing to below threshold luminescence, polarization or transverse mode hopping can be ruled out as the switching mechanism. To model the experiment, we assume that the bottom cavity operates as a laser, with a typical light-current curve of a VCSEL: We treat the response of the top cavity to the injection of laser light from the 
bottom cavity according to the theory of Spencer and Lamb [11]. Solution of the Spencer and Lamb equations for a given injected laser field can lead to single-stable or bistable steady-state solutions, depending on the combination of injected intensity, nonlinear absorption in the top cavity, and detuning between the two cavities. The changes in these quantities are assumed to arise from heating. Figure 3 shows the calculated output intensity from the top cavity as a function of the oxide cavity current. The calculation, which qualitatively reproduces the experimental data shown in Fig. 2, shows a wide bistable region located near the peak of the laser emission. The saturable absorption required for bistability comes from the quantum wells in the top cavity. The theoretical model also allows for changes in detuning between the top and bottom cavities due to differential heating. The inclusion of differential heating is consistent with the observation that the devices generally do not shut off until they are at or near the thermal rollover point on the light-current curve.

. It is also possible to use a bistable CRVCL as a high contrast switching device. Through small changes in the top cavity bias current it is possible to abruptly switch between lasing at $\sim 4 \mathrm{~mW}$ to subthreshold emission at $40 \mu \mathrm{W}$. The left side of Fig. $4 \mathrm{a}$ shows the light-current curve for two different bias currents for a device with an air-post top cavity. The right side of Fig. 4a shows the corresponding emission spectrum on a log scale with the oxide cavity current fixed at $31.5 \mathrm{~mA}$. The top curve shows the multimode lasing spectrum at the longer cavity resonance for a $3.5 \mathrm{~mA}$ top cavity bias and the bottom curve shows emission at $2.5 \mathrm{~mA}$ bias where two broad subthreshold luminescence peaks are observed at the long and short cavity resonances. Thus, it is possible to abruptly change the optical output by $20 \mathrm{~dB}$ by varying the bias current to the top cavity by as little as $1 \mathrm{~mA}$. Similar data is shown in Fig. $4 \mathrm{~b}$ for a device with an implanted top cavity. Since the implant aperture creates a higher current density for a given input current, this device can be turned off and on by changing the top cavity bias by as 
little as $0.1 \mathrm{~mA}$. Thus, changes in electrical power as low as $250 \mu \mathrm{W}$ can change the output state intensity by two orders of magnitude.

In summary, we have observed bistable output from a coupled-resonator vertical-cavity laser diode. We have fabricated devices with an implanted current aperture in the top cavity and an oxide aperture in the bottom cavity which yielded output powers as high as $7 \mathrm{~mW}$. Theoretical calculations based on the method of Spencer and Lamb are shown to qualitatively reproduce the observed light-current curves. The mechanism of the observed bistability was determined to be the nonlinear optical response of the top cavity combined with changes in the intracavity intensity in the bottom cavity due to thermal rollover. High contrast switching from full power lasing to off-state luminescence was also demonstrated using very low electrical switching powers. Further experiments are underway to determine if the CRVCL can also be switched using external optical injection.

- This research was performed at Sandia National Laboratories, a multiprogram laboratory operated by Sandia Corporation, for the United States Department of Energy under contract No. DE-AC04-94AL85000. 


\section{Figure Captions:}

Fig. 1: (a) Schematic of a coupled-resonator vertical cavity laser with two active cavities. Note that an implanted current aperture is used in the top cavity while an oxide aperture is used in the bottom cavity.

Fig. 2 : (a) Bistable light vs. current curve plotted for three different top cavity bias currents. Note the large bistable region extending for more than $18 \mathrm{~mA}$. (b) Bistable light vs. current curve for another device. Note the slightly, smaller bistable regions and the abrupt turn-on at threshold due to the saturation of the top cavity absorption (denoted with an arrow).

Fig. $3^{\prime}$ : Calculated bistable light vs. current curve. Note that the theory reproduces the large bistable region on the high current side of the plot.

Fig. 4 : High contrast switching for a top cavity (a) without an implant and (b) with an implant aperture. The plots on the right show the emission spectrum for two different bias currents applied to the top cavity with a fixed current applied to the oxide cavity. Note the transition from multimode lasing at the long wavelength resonance to subthreshold luminescence from both cavity resonances. 


\section{References:}

[1] For a recent review see H. Kawaguchi, IEEE J. Select Topics Quantum Electron. 3, 1254 (1997).

[2] M.J. Bohn and J.G. McInerney, J. Opt. Soc. Am. B 14, 3430 (1997); F. Jeannés, G. Patriarche, R. Azoulay, A. Ougazzaden, J. Landreau, and J.L. Oudar, IEEE Photon. Tech. Lett. 8, 539 (1996).

[3] X. Tang, J.P. Van der Ziel, B. Chang, R. Johnson, and J.A. Tatum, IEEE J. Quantum Electron. 33, 927 (1997).

[4] S.F. Lim, J.A. Hudgings, G.S. Li, W. Yuen, K.Y. Lau, and C.J. Chang-Hasnain, Electron. Lett. 33, $1708(1997)$.

[5] Z.G. Pan, S. Jiang, M Dagenais, R.A. Morgan, K. Kojima, M.T. Asom, R.E. Leibenguth, G.D. Guth, M.W. Focht, Appl. Phys. Lett. 63, 2999 (1993); H. Kawaguchi, I.S. Hidayat, Y. Takahashi, and T. Yamayoshi, Electron Lett. 31, 109 (1995).

[6] N.K. Dutta, G.P. Agrawal, and M.W. Focht, Appl. Phys. Lett. 44, 30 (183).

[7] K.D. Choquette, W.W. Chow, H.Q. Hou, K.M. Geib, and B.E. Hammons, Proceeding of the SPIEThe International Society for Optical Engineering 3286, 134 (1998); A.J. Fischer, K.D. Choquette, W.W. Chow, H.Q. Hou, and K.M. Geib, Appl. Phys. Lett. 75, 3020 (1999);

[8] A.J. Fischer, W.W. Chow, K.D. Choquette, A.A. Allerman, and K.M. Geib, Appl. Phys. Lett. 76, $1975(2000)$.

[9] J.F. Carlin, R.P. Stanley, P. Pellandini, U. Oesterle, and M. Ilegems, Appl. Phys. Lett. 75, 908 (1999); [10] P. Michler, M. Hilpert, and G. Reiner, Appl. Phys. Lett. 70, 2073 (1997).

[11] M.B. Spencer and W.E. Lamb, Jr., Phys. Rev. A 5, 884 (1972). 
Figure 1, A.J. Fischer et al.

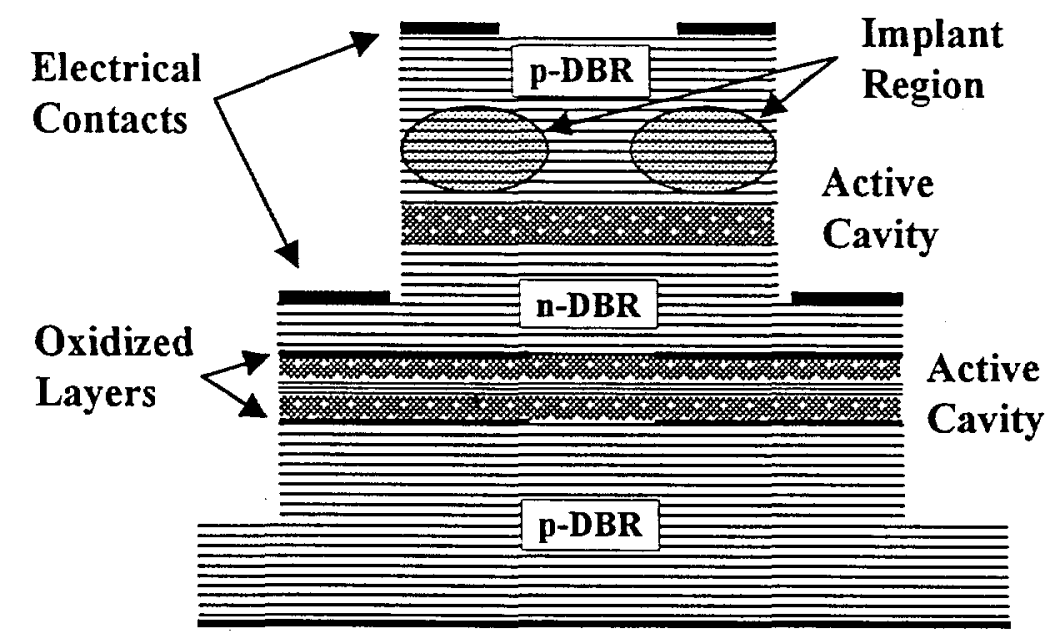



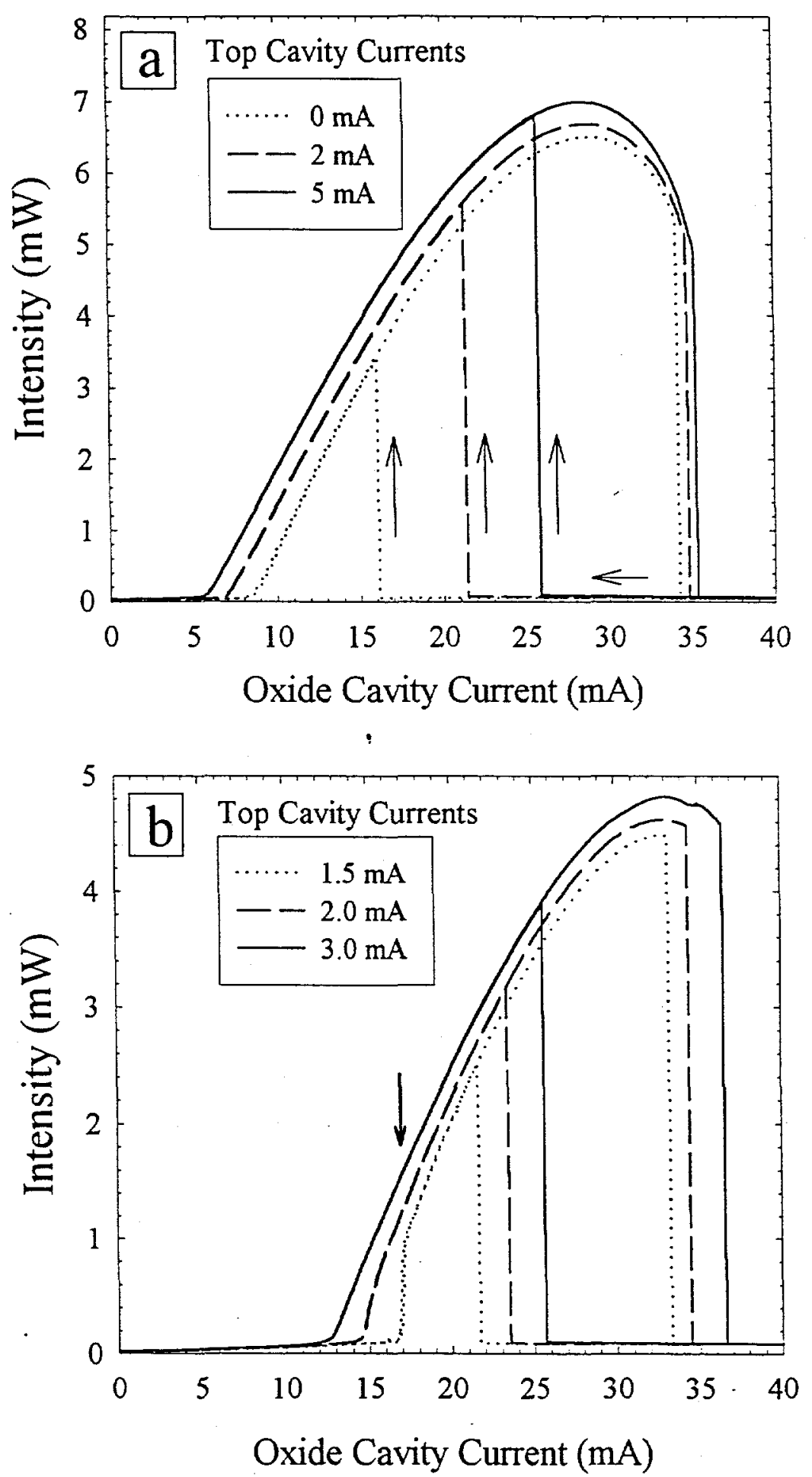


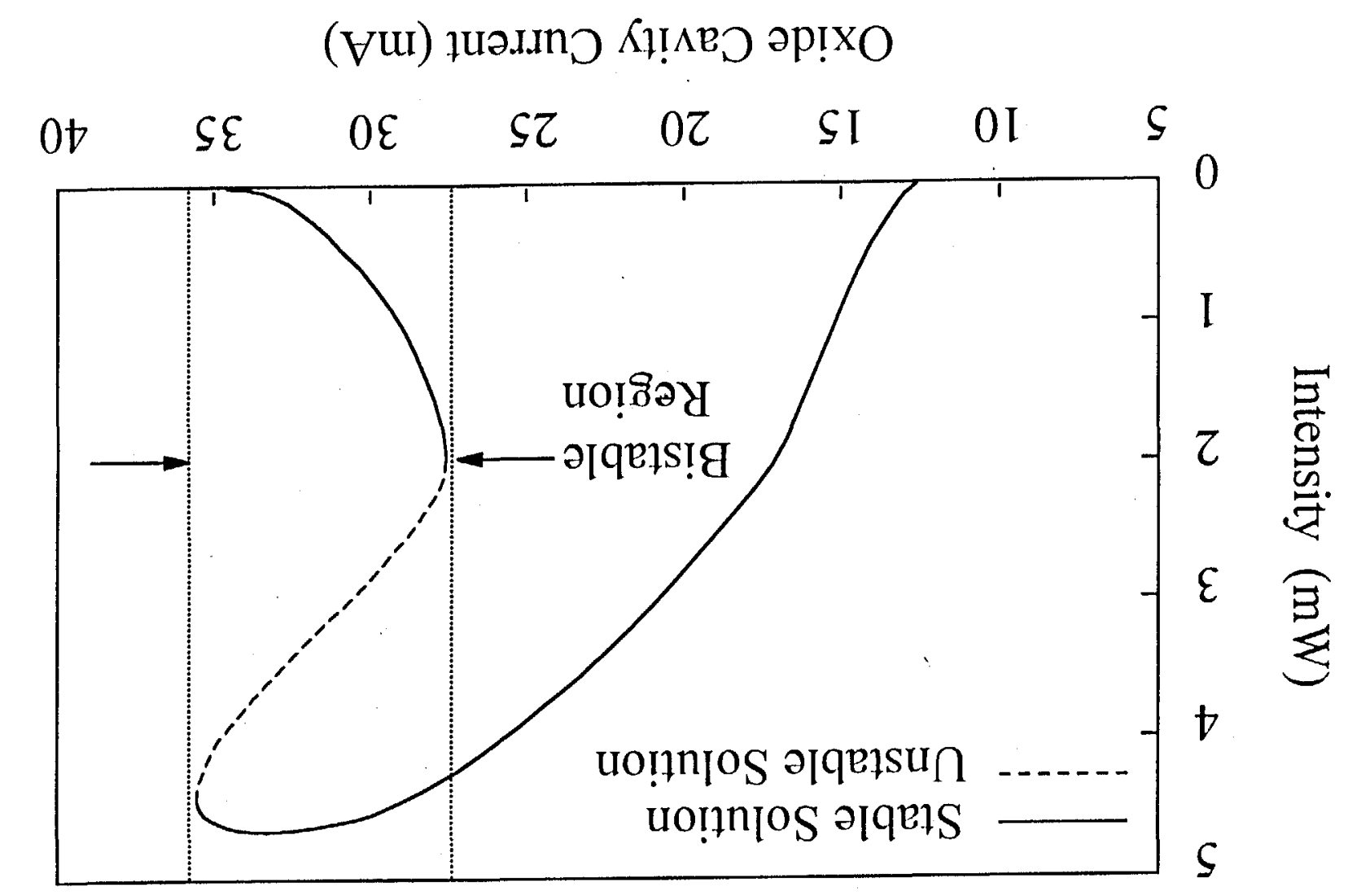



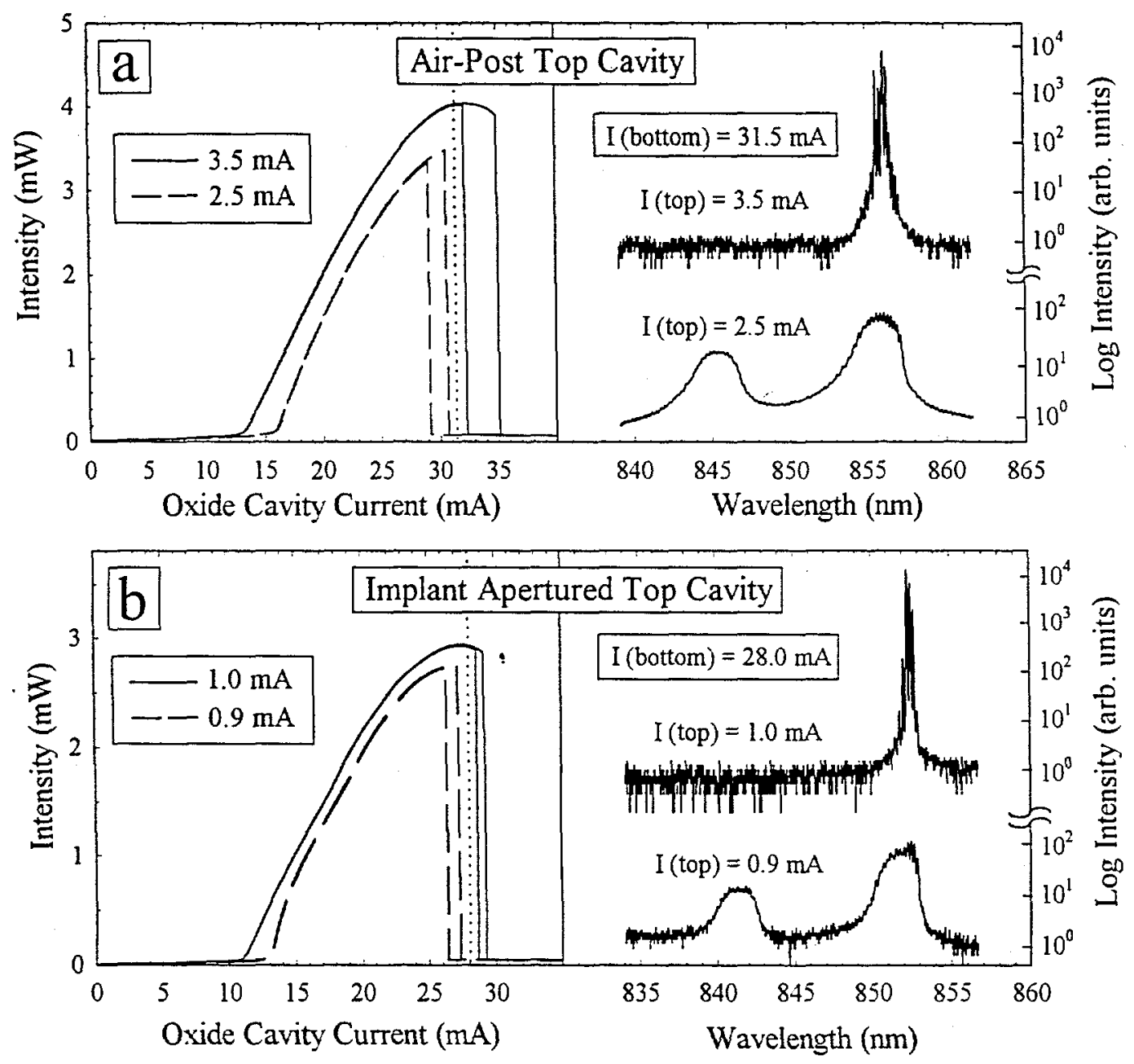